\title{
Higher Serum Lactic Dehydrogenase is Associated with Post-Stroke Depression at Discharge
}

\author{
Guo $\mathrm{Li}^{\prime}$ \\ Jinfeng Miao' \\ Chensheng Pan (1) \\ Ping Jing (iD ${ }^{2}$ \\ Guohua Chen ${ }^{3}$ \\ Junhua $\mathrm{Mei}^{3}$ \\ Wenzhe Sun' \\ Yan Lan $\mathbb{D}^{\prime}$ \\ Xin Zhao' \\ Xiuli Qiu' \\ Yanyan Wang' \\ Zhou Zhu' \\ Suiqiang Zhu' \\ Lifei Lian' \\ 'Department of Neurology, Tongji \\ Hospital, Tongji Medical College, \\ Huazhong University of Science and \\ Technology, Wuhan, Hubei, 430030 , \\ People's Republic of China; ${ }^{2}$ Department \\ of Neurology, Wuhan Central Hospital, \\ Wuhan, Hubei, 4300I4, People's Republic \\ of China; ${ }^{3}$ Department of Neurology, \\ Wuhan First Hospital, Wuhan, Hubei, \\ 430022, People's Republic of China
}

Purpose: Post-stroke depression (PSD) is one of the most common and severe psychological sequelae after stroke, negatively affecting the patient's functional outcome and quality of life. Rapidly measurable biomarkers to predict PSD are pivotal for the optimized care and allocation of healthcare resources. Lactic dehydrogenase (LDH) levels are increased in patients with central nervous system (CNS) disorders such as cerebral infarction and hypoxic-ischemic encephalopathy, which may be related to the occurrence of PSD in acute ischemic stroke (AIS) patients. This study aimed to investigate whether LDH levels on admission are associated with PSD at discharge.

Patients and Methods: A multicenter prospective cohort study was conducted, including all consecutive AIS patients within 7 days after symptom onset from May 2018 to October 2019. According to the distribution of LDH and the number of patients, patients were divided into equal tertiles. PSD was evaluated by DSM-V criteria and the 17-item Hamilton Rating Scale for Depression (HRSD-17) at discharge.

Results: A total of 518 AIS patients were included. The optimal cut-off points of LDH were: lowest tertile (T1) 102-159/L, middle tertile (T2) 160-189 U/L, and upper tertile (T3) 190-520 U/L. A total of 249 patients (48.07\%) were diagnosed with PSD at discharge. After adjusting for potential confounding factors, the odds ratio of T3 PSD was 1.698 (95\% CI, 1.070-2.694, $P=0.025)$, compared with T1.

Conclusion: In summary, LDH serum levels on admission are associated with PSD at discharge. Clinicians should pay more attention to the baseline LDH level in screening for PSD at discharge.

Keywords: post-stroke depression, lactic dehydrogenase, screen, cohort study

\section{Introduction}

Post-stroke depression (PSD) is one of the most common and severe neuropsychiatric sequelae of stroke, ${ }^{1}$ affecting approximately $40 \%$ of acute ischemic stroke (AIS) survivors. ${ }^{2}$ Many studies have suggested that severely impaired social and cognitive functions, adverse recovery of motor function, poor rehabilitation outcome, increased treatment cost, poor quality of life and increased mortality are related to the existence of PSD. ${ }^{3-5}$ Fudala et al evaluated the functional condition of patients 3 years after stroke. They demonstrated that $46.5 \%$ of respondents were diagnosed as having PSD and $9.9 \%$ of patients were diagnosed with severe depression. ${ }^{6}$ Therefore, stroke and stroke-rehabilitation guidelines recommend screening for PSD in clinical practice. ${ }^{7}$ However, because depressive symptoms may be potentially confused with certain typical symptoms of AIS patients, it is difficult to diagnose PSD. ${ }^{8}$ In addition, although the importance of PSD has been 
fully proven and there are validated screening tools, many PSD patients still cannot be diagnosed by busy stroke clinicians. One of the reasons for this is the lack of reliable objective biomarkers of PSD. ${ }^{9}$ Understanding of the predictors for early PSD diagnosis and the exploration of the potential pathophysiological mechanism of PSD still need to be further clarified. ${ }^{10}$ The identification of additional aspects of PSD mechanisms and modifiable risk factors may lead to the formation of more specific therapeutic interventions targeting the most vulnerable people and may help in the development of future policies. ${ }^{11}$

Lactic dehydrogenase (LDH) is an enzyme that is widely distributed in various tissues and cells, including liver, muscle and brain, and is also known as a marker of disease and tissue injury. LDH catalyzes the interconversion of pyruvate and lactate; the latter is used as fuel by diverse tissues under fully aerobic conditions. It provides the oxidative and gluconeogenic substrates required for cellular homeostasis. ${ }^{12}$ In the brain, lactate is a major source of fuel for brain metabolism and is supplied locally from aerobic glycolysis or through the peripheral circulation. ${ }^{13}$ Studies have shown that patients with central nervous system (CNS) disorders such as hypoxic-ischemic encephalopathy, posterior reversible encephalopathy syndrome and acute stroke have elevated LDH levels as well, ${ }^{14-19}$ and the LDH concentrations may be associated with poor outcome. ${ }^{14,16,19}$

The hypothesis of the study is that LDH may serve as a potential biomarker of PSD at discharge, because it represents the cellular and/or tissue damage under hypoxic-ischemic status. Moreover, LDH is easily and rapidly detectable and available in almost every patient on the ward, with low costs. As a result, LDH was selected as the potential biomarker. The purpose of this study was to evaluate the association between baseline LDH and PSD at discharge.

\section{Materials and Methods}

\section{Study Population and Design}

A multicenter prospective cohort study was conducted at Tongji Hospital, Wuhan First Hospital and Wuhan Central Hospital in Wuhan City, Hubei Province, China. The Ethics Committee of Tongji Medical College, Huazhong University of Science and Technology approved the protocol (ID: TJ-IRB20171108). From May 2018 to October 2019, AIS patients were consecutively recruited from these hospitals within 7 days after the onset of stroke. All subjects provided written informed consent in accordance with the Declaration of Helsinki and its later amendments.

The trial registration number is ChiCTR-ROC -17013993. The original protocols used for this post hoc analysis did not include serum LDH as a potential marker. LDH was measured in the clinical laboratories of Tongji Hospital, Wuhan First Hospital and Wuhan Central Hospital according to a standardized protocol. In clinical practice, there are few concerns about the change in odds ratio (OR) values of the PSD risk when the LDH unit changes. Hence, LDH was not considered as a continuous variable in this study. According to the number of patients and the distribution of LDH levels with the highest differences in this study, LDH levels were further divided into tertiles (102-159, 160-189 and 190-520 U/ L). This helped us to observe whether any enhanced performance could be quantified while maintaining the statistical effect of each category.

\section{Inclusion and Exclusion Criteria}

Within 7 days after admission, all suspected AIS patients had the diagnosis confirmed by computed tomography (CT) or magnetic resonance imaging (MRI). The inclusion criteria were as follows: 1) diagnosed AIS patients, aged $\geq 18$ years, including males and females; 2) written informed consent was provided by AIS patients or their authorized family members; and 3) hospital admission within 7 days after stroke onset.

The exclusive criteria were as follows: 1) brain dysfunction caused by other non-vascular causes, such as brain trauma, subdural hematoma, primary brain tumors, metastatic encephaloma or paralysis after seizures; 2) dementia, history of depression (clinical diagnosis or previous treatment history) and/or other psychiatric illness; 3) unable to complete the follow-up; 4) communication problems caused by aphasia, dysarthria, understanding or consciousness disturbance (Mini-Mental State Examination score $<19$ points, in particular the MMSE score of illiterate patients $<17$ points); ${ }^{20} 5$ ) with other concomitant neuropsychiatric diseases, such as Parkinson's disease or epilepsy; 6) suffering from transient ischemic attack (TIA) or subarachnoid hemorrhage; and/or 7) the serum LDH level was not tested on admission.

\section{Data Collection and Follow-Up}

Sociodemographic and clinical characteristics of AIS patients were collected with a standard case report form (CRF) on admission, including age, gender, level of 
education, marital status, body mass index (BMI), history of smoking and drinking, history of diabetes mellitus, hypertension, hyperlipidemia, atrial fibrillation, coronary heart disease, stroke and surgery. Depressive symptoms were measured at discharge. The stroke severity was assessed within 24 hours after hospital admission by welltrained neurologists using the National Institutes of Health Stroke Scale (NIHSS). Barthel Index (BI) scores and modified Rankin Scale (mRS) scores were also collected.

Serum samples were collected at room temperature on admission, then centrifuged at $3500 \mathrm{rev} / \mathrm{min}$ for 10 minutes to measure the levels of serum biochemical indicators. The concentration of serum C-reactive protein (CRP) was measured by standard autoanalyzer techniques with a Roche automatic analyzer (cobas c701) in the clinical laboratories of the three hospitals. Cytokines, including interleukin-1 $\beta$ (IL-1 $\beta$ ), IL-6, IL-10, IL-18, tumor necrosis factor$\alpha$ (TNF- $\alpha)$, brain-derived neurotrophic factor (BDNF) and interferon- $\gamma$ (IFN- $\gamma$ ), were measured using a solid-phase sandwich enzyme-linked immunosorbent assay (ELISA) kit (CUSABIO, China) according to the manufacturer's specifications, by Kindstar Company, Wuhan. In order to minimize assay variance, all samples were analyzed in duplicate on the same day in a random order by professional clinical technicians who were blind to the clinical diagnoses. The intraassay coefficient was less than $5 \%$.

\section{Psychological Measurement}

All psychological evaluations were performed by three experienced psychiatrists who were blind to other clinical and laboratory indicators, after receiving uniform standardized training. The interrater reliability reached an acceptable level. PSD at discharge was diagnosed according to DSM-V criteria (Depressive Disorder Due to Another Medical Condition). The 17-item Hamilton Rating Scale for Depression (HRSD-17) was used to measure the severity of PSD. Patients who met the DSM-V diagnostic criteria and had an HRSD-17 score $>7$ at discharge were regarded as the primary endpoint of the study. AIS patients were divided into the PSD group and non-PSD group according to whether they were diagnosed as having PSD at discharge.

\section{Statistical Analysis}

Results were expressed as medians (interquartile range, IQR) for continuous variables and percentages for categorical variables. The differences between two groups were compared using the Mann-Whitney $U$-test for continuous variables and the chi-squared test for categorical variables. Statistical comparisons among LDH stratification were assessed by the Kruskal-Wallis test for continuous variables and Pearson's chi-squared test for categorical variables. After adjusting for potential confounders of PSD at discharge, the OR values and 95\% confidence intervals (CIs) of PSD risk at discharge were obtained through multivariate adjusted binary logistic regression. Statistical significance was determined as a two-tailed $P$-value less than $0.05(P<0.05)$. There were 424 first-ever stroke patients and 94 recurrent ischemic stroke patients in our study. We also completed a subgroup analysis. All statistical analyses were performed with $\mathrm{R}$ version 3.5.2 (The $\mathrm{R}$ Foundation for Statistical Computing, Vienna, Austria; http://www.r-pro ject.org) and SPSS for Windows, version 22.0 (IBM Corp., Armonk, NY, USA). R packages "PredictABEL", "rms", "Hmisc" and "ggplot2" were applied.

\section{Results}

\section{Baseline Characteristics of All AIS Patients in LDH Tertiles}

From May 2018 to October 2019, the study recruited a total of 1060 hemorrhagic stroke and AIS patients from Tongji Hospital, Wuhan First Hospital and Wuhan Central Hospital, and 124 hemorrhagic stroke patients were excluded.

There were 370 patients who lacked baseline LDH information because the original protocols used for this post hoc analysis did not include LDH. Of the remaining 566 AIS patients, 48 patients failed to be followed up at discharge. In the end, a total of 518 AIS patients were included in the final analysis, including 403 males (77.80\%) and 115 females (22.20\%). Their mean age was $56.85 \pm 10.16$ years (Table 1). All patients were divided into three groups based on the tertiles of LDH levels between 102 and $520 \mathrm{U} / \mathrm{L}$, which ensured that most categories included an adequate number of AIS patients per subgroup (T1, 171 patients; T2, 173 patients; T3, 174 patients). The cut-off values of the LDH levels in the three tertiles were: (T1) 102-159 U/L, (T2) 160-189 U/ L, and (T3) 190-520 U/L. Table 1 summarizes the baseline sociodemographic and clinical characteristics and laboratory indicators of AIS patients by tertiles of LDH level.

Patients in the lowest (T1), middle (T2) and upper tertile (T3) LDH groups showed significant differences in the following characteristics: gender, history of diabetes 
Table I Baseline Characteristics of AIS Patients According to LDH Tertiles

\begin{tabular}{|c|c|c|c|c|c|}
\hline \multirow[t]{2}{*}{ Variables } & \multirow[t]{2}{*}{ All Patients, $n=518$} & \multicolumn{3}{|c|}{ LDH Tertiles } & \multirow[t]{2}{*}{ P-Value } \\
\hline & & $\begin{array}{l}\text { Tertile I, n=I7| } \\
\text { (102-159 U/L) }\end{array}$ & $\begin{array}{l}\text { Tertile 2, n=173 } \\
\text { (160-189 U/L) }\end{array}$ & $\begin{array}{l}\text { Tertile 3, n=I74 } \\
\text { (190-520 U/L) }\end{array}$ & \\
\hline Serum LDH level (U/L), median (IQR) & $173.5(\mid 53.0,202.0)$ & $143.0(133.0,153.0)$ & $173.0(165.0,181.5)$ & $220.0(202.0,247.3)$ & $<0.001$ \\
\hline \multicolumn{6}{|l|}{ Sociodemographic parameters } \\
\hline Age (years), median (IQR) & $59.0(51.0,65.0)$ & $56.0(50.0,65.0)$ & $59.0(50.5,65.0)$ & $61.0(52.0,67.3)$ & 0.177 \\
\hline Females, n (\%) & $115(22.20)$ & $28(16.37)$ & $35(20.23)$ & $52(29.89)$ & 0.008 \\
\hline BMI $\left(\mathrm{kg} / \mathrm{m}^{2}\right)$, median $(\mathrm{IQR})$ & $24.67(22.60,26.79)$ & $24.67(22.79,27.04)$ & $24.51(22.49,26.49)$ & $24.67(22.49,26.65)$ & 0.520 \\
\hline Married, n (\%) & $493(95.17)$ & $161(94.15)$ & $164(94.80)$ & $168(96.55)$ & 0.560 \\
\hline Educational level & & & & & 0.085 \\
\hline Junior middle school and below, $\mathrm{n}(\%)$ & $327(63.13)$ & $100(58.48)$ & $107(61.85)$ & $120(68.97)$ & \\
\hline Senior high/polytechnic school, n (\%) & 116 (22.39) & $39(22.8 \mathrm{I})$ & $38(21.97)$ & $39(22.4 I)$ & \\
\hline Bachelor and above, $\mathrm{n}(\%)$ & $75(14.48)$ & $32(|8.7|)$ & $28(16.18)$ & $15(8.62)$ & \\
\hline \multicolumn{6}{|l|}{ Vascular risk factors } \\
\hline History of smoking, n (\%) & $309(59.65)$ & $114(66.67)$ & $101(58.38)$ & $94(54.02)$ & 0.052 \\
\hline History of drinking, n (\%) & $302(58.30)$ & $103(60.23)$ & $104(60.12)$ & $95(54.60)$ & 0.477 \\
\hline History of diabetes, n (\%) & $133(25.68)$ & $59(34.50)$ & $44(25.43)$ & $30(17.24)$ & 0.001 \\
\hline History of hypertension, n (\%) & $296(57.14)$ & $94(54.97)$ & $107(61.85)$ & $95(54.60)$ & 0.308 \\
\hline History of hyperlipidemia, n (\%) & $122(23.55)$ & $42(24.56)$ & $36(20.81)$ & $44(25.29)$ & 0.574 \\
\hline History of coronary artery diseases, $\mathrm{n}(\%)$ & $51(9.85)$ & $15(8.77)$ & $14(8.09)$ & $22(12.64)$ & 0.308 \\
\hline History of previous stroke, n (\%) & $94(18.15)$ & $31(18.13)$ & $35(20.23)$ & $28(16.09)$ & 0.606 \\
\hline History of atrial fibrillation, $\mathrm{n}(\%)$ & $20(3.86)$ & $3(1.75)$ & $7(4.05)$ & $10(5.75)$ & 0.155 \\
\hline History of surgery, n (\%) & $155(29.92)$ & $60(35.09)$ & $40(23.12)$ & $55(31.6 I)$ & 0.044 \\
\hline \multicolumn{6}{|l|}{ Clinical characteristics } \\
\hline NIHSS score, median (IQR) & $3(1,6)$ & $3(1,5)$ & $3(1,6)$ & $3(2,7)$ & 0.025 \\
\hline BI score, median (IQR) & $90(55,100)$ & $95(65,100)$ & $90(50,100)$ & $82.5(45,100)$ & 0.005 \\
\hline mRS score, median (IQR) & $2(1,4)$ & $2(1,3)$ & $2(1,4)$ & $2(1,4)$ & 0.008 \\
\hline HRSD score, median (IQR) & $6(3,11)$ & $5(2,9)$ & $6(3,11)$ & $7(4,12)$ & 0.001 \\
\hline Hospitalization days, median (IQR) & II $(8,14)$ & $10(8,13)$ & $10(8,13)$ & $12(9,15)$ & 0.003 \\
\hline \multicolumn{6}{|l|}{ Serum biochemistry } \\
\hline CRP (mg/L), median (IQR) & $2.30(0.70,7.25)$ & $1.90(0.55,7.20)$ & $2.10(0.65,7.25)$ & $3.00(0.95,7.25)$ & 0.005 \\
\hline $\mathrm{IL}-\mathrm{I} \beta(\mathrm{pg} / \mathrm{mL})$, median $(\mathrm{IQR})$ & $62.28(26.33,157.21)$ & $53.46(23.77,156.16)$ & $74.25(30.06,167.93)$ & $56.65(23.40,143.77)$ & 0.112 \\
\hline IL-6 (pg/mL), median (IQR) & $6.00(2.43,9.69)$ & $5.59(2.29,8.22)$ & $6.00(2.99,10.11)$ & $6.00(2.37,10.04)$ & 0.353 \\
\hline IL-I0 (pg/mL), median (IQR) & $8.27(2.38,21.58)$ & $8.00(2.25,20.27)$ & $8.76(2.69,27.13)$ & $7.40(1.85,20.87)$ & 0.326 \\
\hline IL-I8 (pg/mL), median (IQR) & $1970.36(957.12,4551.33)$ & $1833.72(1028.52,4646.52)$ & $2143.94(985.27,4035.00)$ & $2121.54(921.43,5119.16)$ & 0.865 \\
\hline TNF- $\alpha$ (pg/mL), median (IQR) & $40.37(23.20,57.36)$ & $41.57(26.30,60.93)$ & $40.64(23.45,55.52)$ & $38.42(20.44,54.72)$ & 0.214 \\
\hline BDNF (ng/mL), median (IQR) & $3.51(2.10,7.11)$ & $3.81(2.42,8.61)$ & $3.36(2.14,7.57)$ & $3.15(1.85,6.86)$ & 0.182 \\
\hline IFN- $\gamma(\mathrm{pg} / \mathrm{mL})$, median (IQR) & $4.33(1.73,9.35)$ & $3.44(1.29,6.99)$ & $4.91(2.25,9.75)$ & $4.23(1.71,8.53)$ & 0.084 \\
\hline
\end{tabular}

and atrial fibrillation, baseline serum CRP level, NIHSS score, BI and mRS scores, hospitalized days and HRSD-17 score at discharge (all $P$-values $<0.05$ ). The patients in the upper LDH tertile (T3) group had the highest HRSD-17 scores. Moreover, there were significant differences in CRP, IL-1 $\beta$, IL-6, IL-10, IL-18, TNF- $\alpha$, BDNF and IFN$\gamma$ between the three groups (all $P$-values $<0.001$ ).

\section{Baseline Characteristics of Patients in the PSD Group and Non-PSD Group}

The comparison of baseline characteristics between the PSD and non-PSD groups is presented in Table 2. A total of 249 patients $(48.07 \%)$ were diagnosed with
PSD at discharge. Compared with the non-PSD group, PSD patients were more likely to have higher serum LDH level, older age, lower educational level, lower BI score, and higher NIHSS, mRS and HRSD scores at discharge, and more hospitalized days. PSD patients also had lower BDNF levels compared with the non-PSD group.

\section{Association Between the Level of LDH} and PSD

Significant differences were also found between the PSD groups and non-PSD groups in the LDH tertiles of patients. The proportions of patients in the lowest tertile $(P=0.002)$ and the upper tertile $(P=0.004$, Table 3$)$ were 
Table 2 Clinical and Sociodemographic Characteristics of Patients with PSD and Non-PSD at Discharge

\begin{tabular}{|c|c|c|c|}
\hline Variables & PSD Patients $(n=249)$ & Non-PSD Patients $(n=269)$ & P-Value \\
\hline Serum LDH level (U/L), median (IQR) & $179.0(158.0,213.0)$ & $168.0(149.0,195.0)$ & 0.001 \\
\hline \multicolumn{4}{|l|}{ Sociodemographic parameters } \\
\hline Age (years), median (IQR) & $61.0(54.0,67.0)$ & $59.0(51.0,65.0)$ & $<0.001$ \\
\hline Females, n (\%) & $57(22.89)$ & $58(21.56)$ & 0.716 \\
\hline BMI $\left(\mathrm{kg} / \mathrm{m}^{2}\right)$, median (IQR) & $24.67(22.86,27.18)$ & $24.67(22.76,26.77)$ & 0.273 \\
\hline Married, n (\%) & 239 (95.98) & $254(94.42)$ & 0.408 \\
\hline Educational level & & & 0.004 \\
\hline Junior middle school and below, n (\%) & $164(65.86)$ & $163(60.59)$ & \\
\hline Senior high/polytechnic school, n (\%) & $62(24.90)$ & $54(20.07)$ & \\
\hline Bachelor and above, $\mathrm{n}(\%)$ & $23(9.24)$ & $52(19.33)$ & \\
\hline \multicolumn{4}{|l|}{ Vascular risk factors } \\
\hline History of smoking, n (\%) & $142(57.03)$ & $167(62.08)$ & $0.24 I$ \\
\hline History of drinking, n (\%) & $151(60.64)$ & $151(56.13)$ & 0.298 \\
\hline History of diabetes, n (\%) & $61(24.50)$ & $72(26.77)$ & 0.555 \\
\hline History of hypertension, $\mathrm{n}(\%)$ & $136(54.62)$ & $160(59.48)$ & 0.264 \\
\hline History of hyperlipidemia, n (\%) & $56(22.49)$ & $66(24.54)$ & 0.584 \\
\hline History of coronary artery diseases, $\mathrm{n}(\%)$ & $30(12.05)$ & $2 I(7.8 I)$ & 0.105 \\
\hline History of previous stroke, $\mathrm{n}(\%)$ & $49(19.68)$ & $45(16.73)$ & 0.384 \\
\hline History of surgery, n (\%) & $73(29.32)$ & $82(30.48)$ & 0.772 \\
\hline History of atrial fibrillation, n (\%) & $8(3.21)$ & $12(4.46)$ & $0.46 \mathrm{I}$ \\
\hline \multicolumn{4}{|l|}{ Clinical characteristics } \\
\hline NIHSS score, median (IQR) & $8(5,10)$ & $2(I, 4)$ & $<0.001$ \\
\hline Bl score, median (IQR) & $95(65,100)$ & $100(80,100)$ & $<0.001$ \\
\hline mRS score, median (IQR) & $4(3,4)$ & $2(I, 3)$ & $<0.001$ \\
\hline HRSD score, median (IQR) & $13(10,18)$ & $3(2,5)$ & $<0.001$ \\
\hline Hospitalization days, median (IQR) & $12(9,15)$ & $9(8,12)$ & $<0.001$ \\
\hline \multicolumn{4}{|l|}{ Serum biochemistry } \\
\hline CRP (mg/L), median (IQR) & $2.40(0.75,7.25)$ & $2.30(0.60,7.25)$ & 0.319 \\
\hline IL-I $\beta$ (pg/mL), median (IQR) & $63.92(30.00,156.16)$ & $62.03(22.86,163.83)$ & 0.623 \\
\hline IL-6 (pg/mL), median (IQR) & $5.75(2.29,9.95)$ & $6.00(2.69,9.37)$ & 0.947 \\
\hline IL-I0 (pg/mL), median (IQR) & $7.76(2.14,21.78)$ & $9.16(2.74,21.60)$ & 0.262 \\
\hline IL-I8 (pg/mL), median (IQR) & $1959.33(951.89,4530.10)$ & I $975.92(968.56,4553.5 \mid)$ & 0.922 \\
\hline TNF- $\alpha(p g / m L)$, median (IQR) & $40.23(22.12,56.01)$ & $40.4 \mathrm{I}(25.22,58.12)$ & 0.433 \\
\hline BDNF (ng/mL), median (IQR) & $3.13(1.94,6.34)$ & $3.82(2.16,8.44)$ & 0.028 \\
\hline IFN- $\gamma(\mathrm{pg} / \mathrm{mL})$, median (IQR) & $4.56(1.89,9.60)$ & $3.86(1.65,8.80)$ & 0.347 \\
\hline
\end{tabular}

both statistically significantly different between PSD and non-PSD groups. In Table 4, the lowest LDH tertile was taken as a reference. We observed the association between LDH and PSD at discharge in the unadjusted and several multivariate adjusted logistic regression models. In the unadjusted logistic regression model, the upper tertile (T3) of LDH could serve as an effective predictor of PSD at discharge. The unadjusted OR value was 2.100 (95\% CI, 1.366-3.288, $P=0.001$ ).

After adjusting for potential confounders including age, gender, educational level, BMI, marital status, history of smoking and drinking, history of coronary artery disease, diabetes mellitus, hyperlipidemia, hypertension, stroke, atrial fibrillation and surgery, baseline NIHSS, mRS and BI scores, hospitalized days, hospital, IL-1 $\beta$, IL-6, IL-10, IL-18, TNF$\alpha$, BDNF, IFN- $\gamma$ and CRP, the upper LDH tertile (T3) remained associated with the prevalence of PSD (for T3, model 1: $\mathrm{OR}=2.007,95 \% \mathrm{CI}, 1.301-3.095, \quad P<0.001$; model 2: $\mathrm{OR}=2.007,95 \% \mathrm{CI}, 1.301-3.095, \quad P<0.001$; model 3: $\mathrm{OR}=1.601,95 \% \mathrm{CI}, 1.002-2.558, P=0.042$; model 4: OR=1.601, 95\% CI, 1.002-2.558, $P=0.042)$ compared with the lowest LDH tertile (T1).

In the subgroup analysis, we divided the baseline LDH levels of 424 first-ever stroke patients into three tertiles: (T1) 102-159/L, (T2) 160-190 U/L, and (T3) 191-520 U/L. After adjustment for potential confounders, the odds ratio of the 
Table 3 Serum LDH Tertiles of Patients

\begin{tabular}{|l|c|c|c|c|}
\hline Variable & $\begin{array}{c}\text { PSD Patients } \\
(\mathbf{n = 2 4 9 )}\end{array}$ & $\begin{array}{c}\text { Non-PSD Patients } \\
(\mathbf{n = 2 6 9 )}\end{array}$ & $\chi^{\mathbf{2}}$ & P-Value \\
\hline Serum LDH level (U/L) & & & 11.595 & 0.003 \\
Tertile I, $\mathrm{n}=171$ (I02-159 U/L) & 66 & 105 & 9.177 & 0.002 \\
Tertile 2, $\mathrm{n}=173(160-189$ U/L) & 84 & 89 & 0.025 & 0.876 \\
Tertile 3, $\mathrm{n}=174$ (190-520 U/L) & 99 & 75 & 8.178 & 0.004 \\
\hline
\end{tabular}

Table 4 Multivariate Adjusted ORs for the Association Between LDH Levels and PSD at Discharge

\begin{tabular}{|c|c|c|c|c|}
\hline & Tertile & $\mathbf{O R}^{\mathrm{a}}$ & 95\% Cl & P-Value \\
\hline \multirow[t]{2}{*}{ Unadjusted } & Middle & 1.502 & $0.978-2.305$ & 0.063 \\
\hline & Upper & 2.100 & $1.366-3.228$ & 0.001 \\
\hline \multirow[t]{2}{*}{ Model I ${ }^{\mathrm{b}}$} & Middle & 1.485 & $0.966-2.284$ & 0.072 \\
\hline & Upper & 2.007 & $\mathrm{I} .30 \mathrm{I}-3.095$ & 0.002 \\
\hline \multirow[t]{2}{*}{ Model $2^{c}$} & Middle & 1.485 & $0.966-2.284$ & 0.072 \\
\hline & Upper & 2.007 & $1.30 \mathrm{I}-3.095$ & 0.002 \\
\hline \multirow[t]{2}{*}{ Model $3^{d}$} & Middle & 1.516 & $0.955-2.406$ & 0.078 \\
\hline & Upper & 1.601 & $1.002-2.558$ & 0.042 \\
\hline \multirow[t]{2}{*}{ Model $4^{\mathrm{e}}$} & Middle & 1.516 & $0.955-2.406$ & 0.078 \\
\hline & Upper & 1.601 & $1.002-2.558$ & 0.042 \\
\hline
\end{tabular}

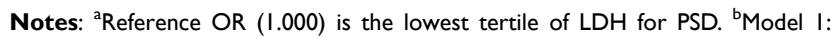
Adjusted for age, gender, educational level, body mass index and marital status. 'Model 2: Adjusted for covariates from model I and further adjusted for history of smoking and drinking, medical history (coronary artery disease, diabetes mellitus, hyperlipidemia, hypertension, previous stroke, atrial fibrillation and surgery). dModel 3: Adjusted for covariates from model 2 and further adjusted for baseline NIHSS scores, mRS scores, Barthel Index scores, hospitalization days and hospital. eModel 4: Adjusted for covariates from model 3 and further adjusted for CRP, IL-I $\beta$, IL-6, IL-10, TNF- $\alpha$, BDNF, IFN- $\gamma$ and IL- 18.

Abbreviations: OR, odds ratio; LDH, lactate dehydrogenase; PSD, post-stroke depression; $\mathrm{Cl}$, confidence interval.

upper tertile (T3) PSD was 1.872 (95\% CI, 1.103-3.176, $P=0.020$ ), compared with the lowest tertile (T1). We also divided the baseline LDH levels of 94 recurrent ischemic stroke patients into three tertiles: (T1) 102-159 U/L, (T2) 160-186 U/L, and (T3) 187-347 U/L. After adjustment for potential confounders, the odds ratio of the upper tertile (T3) PSD was 0.764 (95\% CI, 0.170-3.445, $P=0.727)$, compared with the lowest tertile (T1).

\section{Discussion}

This is the first multicenter prospective cohort study to explore the association between LDH and PSD at discharge in AIS patients. Even after adjusting for potential confounders several times, significant associations were still found between higher baseline LDH and increased risk of PSD at discharge. These findings provide epidemiological evidence that LDH may serve as an effective biomarker for PSD at discharge. Early rehabilitation after discharge is the most important stage of stroke rehabilitation. When clinicians ignore the early onset of PSD, it will be detrimental to the patients' early rehabilitation after discharge. Rapidly measurable biomarkers to predict the development of depression are pivotal for the allocation of health resources and optimized care. ${ }^{21}$ Meanwhile, the identification of measurable biomarkers for depression is also a trend in psychiatric research. Our previous study showed that lower serum uric acid (SUA) was also significantly associated with the risk of PSD at discharge. ${ }^{9}$ Therefore, the findings of this research have major clinical and public implications.

In AIS patients, the underlying mechanism of how LDH associates with PSD remains unclear. Previous studies have shown that a serum LDH test may constitute a useful predictive marker for cellular and/or tissue damage. ${ }^{14-19}$ In this research, a higher serum LDH level was associated with higher NIHSS and mRS scores in AIS patients. Our previous study also demonstrated that increased stroke severity and poor neurological outcome were significantly associated with risk of PSD at discharge. ${ }^{9} \mathrm{LDH}$ is a cytoplasmic enzyme found in the cells of all major organs, which is responsible for converting lactic acid into pyruvic acid, an essential step in producing cellular energy. Normally, astrocytes and neurons in the brain metabolize glucose as the preferred substrate via glycolysis to pyruvate, which is converted to acetyl-CoA to enter the tricarboxylic acid (TCA) cycle. ${ }^{13}$ During stroke, the interrupted or reduced blood flow causes failure of the mitochondrial electron transport chain and oxidative phosphorylation, resulting in ATP depletion, the release of excitatory neurotransmitters and cell death. ${ }^{22}$ Emerging evidence has shown that disturbed energy metabolism, as indicated by perturbations in glycolysis, the TCA cycle and mitochondrial function, is involved in depression. ${ }^{23,24}$ Intracranial stenosis is the most common vascular lesion in Chinese acute stroke patients, ${ }^{25}$ and our previous study demonstrated that cerebral artery stenosis is associated with PSD; the 
hypoperfusion and energy metabolism dysfunction of a specific brain region may be the cause. ${ }^{26}$ Furthermore, data have shown that antidepressant treatments may improve energy metabolism in depression. ${ }^{27,28}$ Based on these reasons, baseline $\mathrm{LDH}$, as an indicator of cellular damage and energy metabolism in ischemic stroke, could potentially predict the risk of PSD at discharge. Moreover, acute stroke may be related to the complications of heart and kidney damage, which may increase the levels of $\mathrm{LDH}$ and mental stress. The use of LDH in predicting PSD may also be related to inflammatory responses, because $\mathrm{LDH}$ has been reported as a promising biomarker for inflammatory burden, and its inhibitors can be used as antiinflammatory agents. ${ }^{29,30}$

Consistent with previous studies, the BDNF level in the PSD group was lower than that in the non-PSD group in our study. ${ }^{31,32}$ Moreover, the AIS patients in the lower tertile of LDH had higher BDNF and lower CRP than the other two groups. A comprehensive overview on the etiology and pathology of PSD indicated that the interrelationships between monoamines, neuroinflammation, stress activation (HPA axis) and neurogenesis are possible primary mechanisms, in which energy metabolism is the common denominator. ${ }^{33}$ In this regard, a pivotal role could be played by BDNF. ${ }^{34}$ This further supports the association between LDH level and PSD at discharge.

Compared with patients without PSD at the time of discharge, patients with PSD had significantly worse functional outcome (higher mRS and lower BI scores), older age and increased stroke severity (higher NIHSS scores, more hospitalized days). This was consistent with previous similar studies. ${ }^{35-37}$ Elderly AIS patients in China may be more likely to have PSD owing to the lack of a wellfunded social security system and healthcare system, cognitive impairment and worsening function. ${ }^{38}$ Higher educational level was potentially associated with a decreased risk of PSD at discharge, probably because it represented a better functional cognitive reserve. ${ }^{39}$

The strengths of this study lie in its prospective nature and multicenter design. Moreover, it included a large sample of AIS patients who completed discharge follow-up. PSD outcome at discharge was measured with a validated objective screening instrument. Since PSD outcome is one of the main priorities in stroke and rehabilitation care, understanding its predictors is highly relevant for clinical practice. Several limitations of this study should also be noted. First, Berkson's bias could not be avoided because this is a hospital-based cohort study, rather than a community-based cohort study, which also limits the generalizability of findings to the entire stroke population. For example, selection bias may be generated as a consequence of excluding patients with a history of depression, and additional large-scale clinical cohort studies are needed to confirm these findings. Second, the rate of loss to follow-up reduced some of the statistical power of the analyses. Third, patients with dysarthria, aphasia and other diseases were excluded. These excluded patients may suffer from depressive symptoms. This may cause a selection bias, resulting in a lower rate of PSD than the actual data. In addition, because the routine laboratory tests did not include testing for the isoenzyme of LDH, we still do not know the definite cause of LDH elevation; therefore, further studies on LDH including an isoenzyme study in AIS patients are needed.

\section{Conclusions}

The diagnosis of depression in the population of stroke patients is more difficult than in other patients. In particular, problems arise in people with anosognosia, aphasia/ dysphasia and other cognitive disorders. The huge focus on PSD in modern research is fully justified because of its negative impact on quality of life, recovery and survival after stroke. Our study suggested that higher levels of $\mathrm{LDH}$ on admission were associated with an increased risk of PSD at discharge. These results can provide important insight into the pathogenesis of PSD. For AIS patients, doctors should pay more attention to the baseline LDH levels in clinical practice to screen PSD patients at discharge. These results should be further confirmed by animal experiments and studies using more representative community-based samples.

\section{Data Sharing Statement}

The data and $\mathrm{R}$ codes that was used to generate the results can be made available upon request from the corresponding author.

\section{Ethics Approval and Informed Consent}

The protocol was approved by the Ethics Committee of Tongji Medical College, Huazhong University of Science and Technology (ID: TJ-IRB20171108). Written informed consent was obtained from all subjects or their caregivers.

\section{Author Contributions}

All authors met the following conditions: 
1. Made a significant contribution to the work reported, whether that is in the conception, study design, execution, acquisition of data, analysis and interpretation, or in all these areas.

2. Drafted or wrote, or substantially revised or critically reviewed, the article.

3. Agreed on the journal to which the article will be submitted.

4. Reviewed and agreed on all versions of the article before submission, during revision, the final version accepted for publication, and any significant changes introduced at the proofing stage.

5. Agreed to take responsibility and be accountable for the contents of the article.

\section{Funding}

This work was financially supported by the National Key R\&D Program of China [grant number 2017YFC1310000], Hubei Technological Innovation Special Fund [grant number 2019ACA132] and National Natural Science Fund of China [grant numbers 82101605 , 82001218]. The funders had no role in the study design, data collection and analysis, or decision to publish.

\section{Disclosure}

All authors declare no competing interests.

\section{References}

1. Rogers SC. Poststroke depression screening: an executive summary. $J$ Neurosci Nurs. 2017;49(2):66-68. doi:10.1097/JNN.0000000 000000270

2. Robinson RG, Jorge RE. Post-stroke depression: a review. Am $J$ Psychiatry. 2016;173(3):221-231. doi:10.1176/appi.ajp.2015.15 030363

3. Rigler SK. Management of poststroke depression in older people. Clin Geriatr Med. 1999;15(4):765-783. doi:10.1016/S0749-0690(18) 30030-2

4. Sugawara N, Metoki N, Hagii J, et al. Effect of depressive symptoms on the length of hospital stay among patients hospitalized for acute stroke in Japan. Neuropsychiatr Dis Treat. 2015;11:2551-2556. doi:10.2147/NDT.S91303

5. Bartoli F, Lillia N, Lax A, et al. Depression after stroke and risk of mortality: a systematic review and meta-analysis. Stroke Res Treat. 2013;2013:862978. doi:10.1155/2013/862978

6. Fudala M, Brola W, Czernicki J. Functional status of patients after stroke depending on neurological and medical complications in three years follow-up. Prz Med Uniw Rzesz Inst Leków. 2013;1:7-20.

7. Dworzynski K, Ritchie G, Playford ED. Stroke rehabilitation: long-term rehabilitation after stroke. Clin Med (Lond). 2015;15 (5):461-464. doi:10.7861/clinmedicine.15-5-461

8. Lipsey JR, Spencer WC, Rabins PV, Robinson RG. Phenomenological comparison of poststroke depression and functional depression. $\mathrm{Am}$ J Psychiatry. 1986;143(4):527-529.
9. Li G, Miao J, Sun W, et al. Lower serum uric acid is associated with post-stroke depression at discharge. Front Psychiatry. 2020;11:52. doi:10.3389/fpsyt.2020.00052

10. Taskaldiran I, Omma T, Onder CE, et al. Neutrophil-to-lymphocyte ratio, monocyte-to-lymphocyte ratio, and platelet-to lymphocyte ratio in different etiological causes of thyrotoxicosis. Turk J Med Sci. 2019;49(6):1687-1692. doi:10.3906/sag-1901-116

11. Filipska K, Wisniewski A, Biercewicz M, et al. Are depression and dementia a common problem for stroke older adults? A review of chosen epidemiological studies. Psychiatr Q. 2020;91(3):807-817. doi:10.1007/s11126-020-09734-5

12. Valvona CJ, Fillmore HL, Nunn PB, Pilkington GJ. The regulation and function of lactate dehydrogenase a: therapeutic potential in brain tumor. Brain Pathol. 2016;26(1):3-17. doi:10.1111/bpa.12299

13. Rho JM. Inhibition of lactate dehydrogenase to treat epilepsy. $N$ Engl $J$ Med. 2015;373(2):187-189. doi:10.1056/NEJMcibr1503558

14. Park JS, You Y, Ahn HJ, et al. Cerebrospinal fluid lactate dehydrogenase as a potential predictor of neurologic outcomes in cardiac arrest survivors who underwent target temperature management. J Crit Care. 2020;57:49-54. doi:10.1016/j.jcrc.2020.02.001

15. Vargas M, Servillo G, Striano P. Serum lactate dehydrogenase as early marker of posterior reversible encephalopathy syndrome: keep your eyes open. Anaesth Intensive Care. 2012;40(3):570-571.

16. Chu H, Huang C, Dong J, et al. Lactate dehydrogenase predicts early hematoma expansion and poor outcomes in intracerebral hemorrhage patients. Transl Stroke Res. 2019;10(6):620-629. doi:10.1007/ s12975-019-0686-7

17. Lampl Y, Paniri Y, Eshel Y, Sarova-Pinhas I. Cerebrospinal fluid lactate dehydrogenase levels in early stroke and transient ischemic attacks. Stroke. 1990;21(6):854-857. doi:10.1161/01.STR.21.6.854

18. Thoresen M, Liu X, Jary S, et al. Lactate dehydrogenase in hypothermia-treated newborn infants with hypoxic-ischaemic encephalopathy. Acta Paediatr. 2012;101(10):1038-1044. doi:10.1111/j.1651-2227.2012.02778.x

19. Anan M, Nagai Y, Fudaba H, Fujiki M. Lactate and lactate dehydrogenase in cistern as biomarkers of early brain injury and delayed cerebral ischemia of subarachnoid hemorrhage. J Stroke Cerebrovasc Dis. 2020;29 (5):104765. doi:10.1016/j.jstrokecerebrovasdis.2020.104765

20. Katzman R, Zhang MY, Qu O-Y, et al. A Chinese version of the Mini-Mental State Examination; impact of illiteracy in a Shanghai dementia survey. $J$ Clin Epidemiol. 1988;41(10):971-978. doi:10.1016/0895-4356(88)90034-0

21. Tu WJ, Dong X, Zhao SJ, Yang DG, Chen H. Prognostic value of plasma neuroendocrine biomarkers in patients with acute ischaemic stroke. J Neuroendocrinol. 2013;25(9):771-778. doi:10.1111/jne.12052

22. Fieschi C, Di Piero V, Lenzi GL, et al. Pathophysiology of ischemic brain disease. Stroke. 1990;21(12 Suppl):IV9-11.

23. Shao WH, Chen JJ, Fan SH, et al. Combined metabolomics and proteomics analysis of major depression in an animal model: perturbed energy metabolism in the chronic mild stressed rat cerebellum. OMICS. 2015;19(7):383-392. doi:10.1089/omi.2014.0164

24. CzarnyP, WignerP, Galecki P, et al. The interplay between inflammation, oxidative stress, DNA damage, DNA repair and mitochondrial dysfunction in depression. Prog Neuropsychopharmacol Biol Psychiatry. 2018;80(Pt C):309-21. doi:10.1016/j.pnpbp.2017.06.036

25. Huang YN, Gao S, Li SW, et al. Vascular lesions in Chinese patients with transient ischemic attacks. Neurology. 1997;48(2):524-525. doi:10.1212/WNL.48.2.524

26. Qiu X, Miao J, Lan Y, et al. Association of cerebral artery stenosis with post-stroke depression at discharge and 3 months after ischemic stroke onset. Front Psychiatry. 2020;11:585201. doi:10.3389/ fpsyt.2020.585201

27. Mills EG, Izzi-Engbeaya C, Abbara A, Comninos AN, Dhillo WS. Functions of galanin, spexin and kisspeptin in metabolism, mood and behaviour. Nat Rev Endocrinol. 2021;17:97-113. doi:10.1038/ s41574-020-00438-1 
28. Huang W, Hu W, Cai L, et al. Acetate supplementation produces antidepressant-like effect via enhanced histone acetylation. J Affect Disord. 2021;281:51-60. doi:10.1016/j.jad.2020.11.121

29. Miyoshi N, Tanigawa T, Nishioka S, et al. Association of salivary lactate dehydrogenase level with systemic inflammation in a Japanese population. J Periodontal Res. 2018;53:487-494. doi:10.1111/jre.12537

30. Manerba M, Di Ianni L, Govoni M, Roberti M, Recanatini M, Di Stefano G. Lactate dehydrogenase inhibitors can reverse inflammation induced changes in colon cancer cells. Eur J Pharm Sci. 2017;96:37-44. doi:10.1016/j.ejps.2016.09.014

31. Cherubini A, Ruggiero C, Polidori MC, Mecocci P. Potential markers of oxidative stress in stroke. Free Radic Biol Med. 2005;39 (7):841-852. doi:10.1016/j.freeradbiomed.2005.06.025

32. Zhang E, Liao P. Brain-derived neurotrophic factor and post-stroke depression. J Neurosci Res. 2020;98(3):537-548. doi:10.1002/ jnr. 24510

33. Ferrari F, Villa RF. The neurobiology of depression: an integrated overview from biological theories to clinical evidence. Mol Neurobiol. 2017;54(7):4847-4865. doi:10.1007/s12035-016-0032-y

34. Burkhalter J, Fiumelli H, Allaman I, Chatton JY, Martin JL. Brainderived neurotrophic factor stimulates energy metabolism in developing cortical neurons. $J$ Neurosci. 2003;23(23):8212-8220. doi:10.1523/JNEUROSCI.23-23-08212.2003
35. Zhang Y, Cheng L, Chen Y, Yang GY, Liu J, Zeng L. Clinical predictor and circulating microRNA profile expression in patients with early onset post-stroke depression. $J$ Affect Disord. 2016;193:51-58. doi:10.1016/j.jad.2015.12.061

36. de Man-van GJ, Hafsteinsdottir TB, Lindeman E, Ettema RG, Grobbee DE, Schuurmans MJ. In-hospital risk prediction for post-stroke depression: development and validation of the Post-stroke Depression Prediction Scale. Stroke. 2013;44 (9):2441-2445. doi:10.1161/STROKEAHA.111.000304

37. Linden T, Blomstrand C, Skoog I. Depressive disorders after 20 months in elderly stroke patients: a case-control study. Stroke. 2007;38(6):1860-1863.

38. De Ryck A, Brouns R, Geurden M, Elseviers M, De Deyn PP, Engelborghs S. Risk factors for poststroke depression: identification of inconsistencies based on a systematic review. J Geriatr Psychiatry Neurol. 2014;27(3):147-158. doi:10.1177/0891988714527514

39. Arauz A, Rodriguez-Agudelo Y, Sosa AL, et al. Vascular cognitive disorders and depression after first-ever stroke: the Fogarty-Mexico Stroke Cohort. Cerebrovasc Dis. 2014;38(4):284-289. doi:10.1159/ 000366471
Clinical Interventions in Aging

\section{Publish your work in this journal}

Clinical Interventions in Aging is an international, peer-reviewed journal focusing on evidence-based reports on the value or lack thereof of treatments intended to prevent or delay the onset of maladaptive correlates of aging in human beings. This journal is indexed on PubMed Central, MedLine, CAS, Scopus and the Elsevie
Dovepress

Bibliographic databases. The manuscript management system is completely online and includes a very quick and fair peer-review system, which is all easy to use. Visit http://www.dovepress.com/ testimonials.php to read real quotes from published authors. 